\title{
The contribution of behavioural science to primary care research: development and evaluation of behaviour change interventions
}

\section{Stephen Sutton}

Professor of Behavioural Science, University of Cambridge, Institute of Public Health, Cambridge, UK

\begin{abstract}
Behavioural science is concerned with predicting, explaining and changing behaviour. Taking a personal perspective, this article aims to show how behavioural science can contribute to primary care research, specifically in relation to the development and evaluation of interventions to change behaviour. After discussing the definition and measurement of behaviour, the principle of compatibility and theories of behaviour change, the article outlines two examples of behaviour change trials (one on medication adherence and the other on physical activity), which were part of a research programme on prevention of chronic disease and its consequences. The examples demonstrate how, in a multidisciplinary context, behavioural science can contribute to primary care research in several important ways, including posing relevant research questions, defining the target behaviour, understanding the psychological determinants of behaviour, developing behaviour change interventions and selection or development of measures. The article concludes with a number of recommendations: (i) whether the aim is prediction, explanation or change, defining the target behaviour is a crucial first step; (ii) interventions should be explicitly based on theories that specify the factors that need to be changed in order to produce the desired change in behaviour; (iii) intervention developers need to be aware of the differences between different theories and select a theory only after careful consideration of the alternatives assessed against relevant criteria; and (iv) developers need to be aware that interventions can never be entirely theory based.
\end{abstract}

Key words: behaviour change; interventions; primary care; theories; trials

Received 29 September 2010; accepted 28 March 2011; first published online 22 June 2011

\section{Introduction}

Behavioural science is concerned with predicting, explaining and changing behaviour. In primary care settings, the behaviours of interest may be those of staff or patients. Examples of relevant behaviours are, for patients, attending for appointments, asking for advice about symptoms

Correspondence to: Dr Stephen Sutton, General Practice \& Primary Care Research Unit, University of Cambridge, Institute of Public Health, Forvie Site, Robinson Way, Cambridge CB2 0SR, UK. Email: srs34@medschl.cam.ac.uk and taking medication, and for staff, assessing cardiovascular risk, explaining treatment options and giving health promotion advice. The distinction between prediction and explanation is an important one (Sutton, 1998). In the simplest case, prediction means identifying an association between one variable $(X)$ measured at one time point and another variable ( $Y$; in this case, a behaviour), measured at a later time point. Identifying such an association may be useful even if $X$ is not a cause of $Y$. For example, if we had a simple questionnaire measure that predicted, with reasonable accuracy, whether an adolescent would become a 
smoker in the next five years, this would allow us to identify those adolescents at higher risk and to intervene in some way to prevent the uptake of smoking. By contrast, explanation means identifying causes of the behaviour. Identifying causes, rather than simply predictors, of a behaviour is ultimately more useful because in principle it informs us how the behaviour might be changed: interventions can be designed to target the determinants of the behaviour that are amenable to change (Sutton, 2010).

In this article, I will draw mainly on my own work and that of my collaborators to show how behavioural science can contribute to primary care research, specifically in relation to the development and evaluation of interventions to change behaviour. It is important to acknowledge at the outset that, although they may take a distinctive approach to the topic, the study of behaviour is not the exclusive domain of psychologists. Scientists from other disciplinary backgrounds also study the causes and consequences of behaviour. For example, epidemiologists are interested in behaviours as exposures or risk factors for disease, and share a concern with the accurate measurement of behaviour. In my work, I am fortunate in being a member of several multidisciplinary research teams that include primary care physicians, social scientists, statisticians, epidemiologists and health economists, as well as other psychologists and I am convinced that the quality and the relevance of the research are enhanced by such collaboration.

I will start by discussing the definition and measurement of behaviour and then discuss two examples of behaviour change trials (one on medication adherence and the other on physical activity), which were part of the University of Cambridge General Practice \& Primary Care Research Unit's research programme on prevention of chronic disease and its consequences.

\section{Definition and measurement of behaviour}

Whether the aim is prediction, explanation or change, defining the behaviour of interest is a crucial first step and one that is often not given sufficient attention.

Most studies use self-report measures of behaviour. Following Ajzen and Fishbein (1980), behaviours can be defined in terms of four components: action, target, time and context. The action component is a necessary part of the definition of any behaviour. The target component is usually necessary, though not always. Time and context are optional; they enable the definition of the behaviour to be as specific as required. For example, consider the definition 'eat breakfast tomorrow'. Here, 'eat' is the action, 'breakfast' is the target (alternatives would be 'a bowl of cereal' or 'lunch') and 'tomorrow' is the time component. No context is specified in this example. As an illustration of the importance of context, consider the following definitions:

1) using a condom the next time I have sex;

2) using a condom the next time I have sex with my regular partner;

3) using a condom the next time I have sex with a new sexual partner.

The first definition omits a potentially important contextual factor, namely type of partner, and is therefore probably too general for most purposes. The other two definitions each specify a context. These may be considered to be quite different behaviours both from a public health viewpoint and from the standpoint of the individual who has both types of sexual partner.

Some behaviours are defined so generally that they are best thought of as behavioural categories (Ajzen and Fishbein, 1980). Behavioural categories cannot be directly observed. Instead, they are inferred from single actions assumed to be instances of the general behavioural category. For example, dieting may be inferred from specific behaviours such as eating two instead of three meals a day, not eating desserts, drinking tea and coffee without adding sugar, taking diet pills, etc.

The limitations of self-report measures are well known (eg, Johnston et al., 2004), but in many cases there will be no feasible alternative. Sometimes, it may be possible to use 'objective' measures of behaviour, but these usually have limitations too. For example, cotinine concentration (in saliva, urine or plasma), which is the gold standard method for biochemical validation of self-reported abstinence from smoking, is sensitive only to recent intake of tobacco smoke and cannot be used if the smoker is still using nicotine replacement. 


\section{The principle of compatibility}

The principle of compatibility (Ajzen, 1988) states that, in order to maximise prediction, the target behaviour and its putative determinants should be measured at the same level of specificity. For example, in the context of a workplace smoking cessation intervention, a person's attitude towards stopping smoking may not necessarily be very predictive of whether they stop smoking at work. Predictor and criterion should be closely matched. The principle can be extended beyond prediction to explanation and change (Sutton, 1998). To continue with the same example, an intervention that aimed to encourage employees not to smoke at work should target the determinants of this specific behaviour.

\section{Theories}

Behavioural science has a plethora of theories. To people from other disciplines, many of these theories look very similar to one another. However, just as Eskimos are said to be able to distinguish between 17 different varieties of snow, behavioural scientists can appreciate the subtle differences between different theories. Theories of health behaviour can be classified in a number of ways. It is useful to specify the range of application of a theory. General theories, such as the theory of planned behaviour (TPB; Ajzen, 1991), are applicable to a wide range of behaviours, and not simply health-related behaviours. General theories use the same limited set of constructs to explain behaviours as diverse as why some people lie about their tax returns and others do not, why some people choose a career in medicine while others follow alternative pathways and why some adolescents binge drink more often than others. Other theories are specific to health-related behaviours; for example, the health belief model (Strecher and Rosenstock, 1997) was originally developed to explain why some people use health services such as screening programmes.

Both the TPB and the health belief model focus on individuals' beliefs about the behaviour as important predictors and determinants of that behaviour. Another widely used theory, variously referred to as the common sense, self-regulation or illness representations model (Leventhal et al., 1984), focuses more on individuals' beliefs about an illness - either an illness that the person already has or one that he or she may be at risk of developing. The evidence suggests that theories that focus on beliefs about the behaviour predict behaviour better than those that focus on beliefs about the illness (eg, Hagger and Orbell, 2003).

Another important distinction is between stage and non-stage (or continuum) theories (Weinstein et al., 1998). Unlike theories such as the TPB and the health belief model, stage theories assume that behaviour change involves movement through a sequence of discrete stages, different factors are important at different stages, and therefore that different (stage-matched) interventions should be used for people in different stages. The best known stage theory is the transtheoretical model (Prochaska and Velicer, 1997), sometimes simply referred to as the stages of change model, which has been applied to a wide range of health-related behaviours. However, although the idea of stages of behaviour change is interesting and appealing, this particular model suffers from serious conceptual and measurement problems and cannot be recommended in its present form (Sutton, 2001; West, 2005).

Although mystique surrounds theories in behavioural science, 'there is nothing so practical as a good theory' (Lewin, 1951: p. 169). Theories are useful in intervention development because they specify determinants of behaviour that are potentially amenable to change. Thus, theories inform us which variables should be targeted in interventions - which variables we should try to change in order to produce the desired change in behaviour. These are also the variables that we should measure (along with the target behaviour) when evaluating the impact of an intervention. In particular, it is important to assess whether an intervention that is designed to target behavioural determinants such as self-efficacy or attitude actually produces changes in these variables. If it does not, this provides one plausible reason for a lack of an intervention effect on behaviour.

In the two examples outlined below, we used the TPB to inform the interventions. Although there are some complexities in applying this theory (Sutton, 2010), we chose it because it has several advantages over alternative theories. First, it is a general theory. Stroebe argues that general theories should be preferred on grounds of 
parsimony 'it is not very economical to continue to entertain specific theories of health behaviour unless the predictive success of these models is greater than that of general models of behaviour' (Stroebe, 2000: p. 27). Second, in the TPB, the constructs are clearly defined and the causal relationships between the constructs are clearly specified. Third, there exist clear recommendations for how the constructs should be operationalised (Ajzen, 2006). Fourth, the theory has been widely used to study health behaviours (Ogden, 2003). Finally, meta-analyses of observational studies show that it accounts for a useful amount of variance in intentions and behaviour (but see Sutton (2004) for a discussion of effective variance explained).

\section{Example 1: Medication adherence}

It has been estimated that between a third and a half of medicines for long-term conditions are not taken as prescribed (World Health Organisation, 2003). Among patients with type 2 diabetes who continue to take medication, only about $70-80 \%$ of doses are taken as prescribed (Cramer, 2004). Failure to take medication has serious consequences, including increasing morbidity and wasting health-care resources. There is a need for more effective interventions to support medication adherence. Ideally, these should be brief and capable of being delivered by primary care staff. In the SAMS study (Support and Advice for Medication), we drew on theory and evidence from behavioural science to develop an intervention that targets the determinants of non-adherence. These can be classified as intentional and non-intentional non-adherence (Barber, 2002). Intentional nonadherence refers to non-adherence that arises from the patient's decision to take less medication than prescribed or to miss a dose or a day of medication, perhaps because he or she is concerned about possible side effects or is not convinced that it is necessary to take a full dose of medication in order to derive some benefit. Non-intentional nonadherence refers to forgetting to take medication in spite of an intention to do so.

In the context of oral hypoglycaemic medication for type 2 diabetes, we therefore developed an intervention consisting of two components: a motivational component designed to address intentional non-adherence and a volitional component designed to address non-intentional nonadherence. The motivational component was based on the TPB and was informed by a study of beliefs about medication that was guided by this theory (Farmer et al., 2006). The practice nurse was asked to elicit the patient's salient beliefs about taking medication using open-ended questions such as 'What do you think would be the advantages for you of taking your diabetes medicine(s) regularly?' and 'What do you think makes (would make) it difficult for you to take your medication regularly?'. According to the TPB, those beliefs that are 'top of the mind' and are elicited first in response to such open-ended questions are the beliefs that determine people's intentions and behaviour. The nurses were taught to reinforce positive beliefs both verbally (eg, 'Yes, the diabetes tablets help you to control blood glucose level') and non-verbally (eg, by nodding or smiling) and to 'problem solve' negative beliefs, for example by gently correcting a misconception.

In the volitional component of the intervention, the participant was asked to write down a plan that specifies where, when and how they will take their tablets. These specific action plans are called 'implementation intentions'. The theory is that forming an implementation intention increases the likelihood that the person notices the situation or 'cue' specified in the plan and that the appropriate behavioural response is elicited. An example would be 'If I switch on the kettle in the kitchen in the morning, then I will take my bottle of tablets from the shelf above, open it and take one.' A systematic review showed that implementation intention interventions have mediumto-large effects on health behaviours (Gollwitzer and Sheeran, 2006), although several more recent and well-designed studies have shown no effect (eg, Rutter et al., 2006). Theoretically, implementation intentions should be more effective if people already have a strong goal intention (in this case to take medicine as prescribed), and there is some supportive evidence for this hypothesis (Prestwich and Kellar, in press). This provides a further justification for combining motivational and volitional components, although if strong motivation could be assumed, the intervention could be simplified by dropping the motivational component. 
We tested the effectiveness of this intervention as delivered by practice nurses in a single consultation (Farmer et al., 2008; Farmer et al., submitted for publication). A total of 211 adults with type 2 diabetes in 13 UK practices were randomised to intervention or standard care. The findings were encouraging. The percentage of adherent days, measured objectively with an electronic medication-monitoring device, was significantly higher in the intervention group compared with the standard care group $(77.4 \%$ versus $69.0 \%)$; the difference was equivalent to about one week over the 12 weeks of the trial. There was no significant adverse impact on function or treatment satisfaction. There was no difference between groups in $\mathrm{HbA}_{1 \mathrm{c}}$ but the follow-up may have been too short to detect an effect on this outcome.

In behaviour change trials, the intervention is often an undescribed 'black box'. Even when there is a detailed intervention protocol, it is not always clear to what extent the intervention deliverers adhered to the protocol and what went on in the consultations. SAMS was designed to enable these issues to be investigated. The great majority of the consultations were audiotaped, and analysis of the transcripts is under way. To try to understand the possible mechanism of effect, we also measured potential mediators such as attitudes, intentions and habits. Preliminary analyses suggest that there were no differences between groups on these measures. This may be due to imprecise measurement of mediators or it may indicate that the intervention had its effect via other pathways.

\section{Example 2: Physical activity}

Physical inactivity is a major public health problem (Murray and Lopez, 1996), but evidence for the effectiveness of interventions targeting individuals is mixed. A review of randomised and non-randomised studies concluded that brief advice by primary care practitioners showed promise, but there was uncertainty about the most effective approaches among high-risk groups (National Institute for Health and Clinical Excellence, 2006). The ProActive trial (Williams et al., 2004; Kinmonth et al., 2008) evaluated the efficacy of a theory-based intervention aimed at increasing physical activity, over and above a theory-based brief advice leaflet, among sedentary adults with a parental history of type 2 diabetes, who are at risk of developing diabetes. They were recruited via general practice clinics in the East of England. Highly active people were excluded. In all, 365 participants were randomised to 1) a brief leaflet containing advice to increase physical activity and a persuasive message emphasising benefits (comparison); 2) the leaflet plus an intervention programme delivered at participants' homes (face-to-face); or 3) the leaflet plus the same intervention programme delivered by phone (telephone). The programme aimed to increase physical activity by targeting cognitive mediators based on the TPB. The intervention was delivered by trained facilitators from a range of health professions. Participants had intensive biochemical, anthropometric, behavioural and psychological assessment at baseline and 12 months, and behavioural and psychological assessment at six months. Unlike most previous trials of physical activity interventions, ProActive used objective as well as self-report measures of physical activity.

The results showed that the combined intervention groups did not differ significantly from the comparison group with regard to objective physical activity (primary outcome), self-reported physical activity or cardiorespiratory fitness at 12 months (secondary outcomes) or self-reported physical activity at six months. Face-to-face delivery showed no advantage over telephone delivery.

The obvious question raised by the findings from ProActive is 'Why didn't the intervention work?' or more precisely 'Why was there no difference between the intervention conditions?'. As with SAMS, we included measures of potential mediators of the intervention effect. If an intervention shows no effect, one explanation is that the intervention failed to change the mediators. At six months, the intervention groups showed stronger agreement with the 'affective attitude' items (eg, 'for me, being more physically active in the next 12 months would be enjoyable') and the 'instrumental attitude' items (eg, 'being more physically active in the next 12 months would be good for me'), compared with the advice-only group (Hardeman et al., 2009). They also perceived more control over increasing physical activity over the next 12 months and had stronger intentions to do. Of these, intention showed the 
largest effect: the standardised difference in means was 0.50 , which is a 'medium' effect according to widely used guidelines (Cohen, 1992). In absolute terms, the difference was equal to about one-third of a scale point on a 1-5 rating scale. This may not have been sufficient to produce a difference in behaviour six months later.

There are other possible reasons for the negative findings. The intervention may have been ineffective because we did not include potentially effective behaviour change techniques such as giving participants specific behavioural targets, unlike the Diabetes Prevention Program for example (Diabetes Prevention Program Research Group, 2002). The intervention was complex, and interaction between its components may have reduced its effectiveness. The intervention may not have targeted the important mediators of physical activity change. In the ProActive trial cohort, the TPB cognitions did not predict selfreported and objective physical activity levels or change (Hardeman et al., in press).

Another possible explanation for the lack of intervention effect is that the intervention was not delivered fully as intended. Detailed analysis of sessions with $10 \%$ of the intervention participants showed that the facilitators applied about half of the behaviour change techniques specified in the protocols (Hardeman et al., 2008). This may have been an insufficient 'dose' to help participants to translate their intention into behaviour change.

Although there was no intervention effect, the ProActive trial cohort showed an increase in activity equivalent to $20 \mathrm{~min}$ brisk walking per day on average over the year (Kinmonth et al., 2008). This may have been due to the effects of the advice leaflet, intensive measurement or trial participation, and may have pre-empted an intervention effect. Physical activity measurement included a treadmill test and wearing a heart rate monitor, which may have increased awareness of activity levels and facilitated behaviour change without influencing the TPB cognitions measured. This explanation for the lack of an intervention effect assumes that the amount by which physical activity can feasibly be increased is very limited. If these factors (leaflet, measurement, trial participation), which were common to all three groups, produced an increase in physical activity, the intervention may not have been able to produce a further increase.
Given that we observed a cohort effect, we were also interested in identifying who increased their physical activity. We examined a wide range of baseline predictors, including age, sex and socioeconomic status; TPB measures; body mass index (BMI); clinical variables (such as blood pressure and cholesterol); and psychosocial variables such as self-reported anxiety and health status (Simmons et al., 2010). Some of these variables may be determinants of increases in physical activity, whereas others may not be causally related to change but, if shown to be predictive, could be used to improve targeting of interventions. For example, if on average subgroup A increases their physical activity and subgroup B does not, this might suggest that a different approach needs to be developed for the latter subgroup. In the event, we found few significant independent predictors of behaviour change. Men were more physically active at baseline than women and increased their physical activity more over the 12-month follow-up period, suggesting that a different intervention approach may be needed for women. In addition, participants who were more physically fit at baseline increased their physical activity more, perhaps because it was easier for them to do so. To improve our understanding of the factors influencing change in physical activity, future research should investigate other categories of potential determinants such as environmental and social factors. See Sutton (2004) for a discussion of how theories such as the TPB can be extended into a broader theoretical framework, drawing on the social ecological framework (eg, McLeroy et al., 1988) and ideas from multilevel modelling.

\section{Conclusions and recommendations}

The two examples discussed above demonstrate how, in a multidisciplinary context, behavioural science can contribute to primary care research in several important ways, including posing relevant research questions, defining the target behaviour, understanding the psychological determinants of behaviour, developing behaviour change interventions and selection or development of measures.

I will conclude by making a number of recommendations about the definition of the target behaviour and the use of theory in the development of behaviour change interventions. 
First, defining the target behaviour is a key step. Relevant criteria include the clinical and health outcomes of the behaviour and availability of good measures. The target population also needs to be taken into account. For example, we targeted physical activity in ProActive because the epidemiological evidence suggested that activity levels are more important than diet in the aetiology of type 2 diabetes and that sedentary lifestyle interacts with family history of diabetes in predicting future diabetes risk and because state-of-the-art objective measures of physical activity were available. We focused on increasing moderate rather than strenuous physical activity because it is more feasible and more acceptable to participants.

Second, interventions should be explicitly based on theory. The rationale for this recommendation is straightforward: theories of behaviour change identify the factors that need to be changed in order to produce the desired change in behaviour. A theory of behaviour change can be embedded in a larger causal model that specifies the hypothesised causal relationships between the components of the proposed intervention (including the proposed behaviour change techniques), the determinants of the target behaviour, the behaviour itself and consequent clinical and health outcomes (Hardeman et al., 2005). Drawing a causal model is a useful early step in the process of planning and designing an intervention. Such a model also helps in designing the evaluation of the intervention. In particular, it informs decisions about what should be measured; for example, measures of the theoretical determinants of the target behaviour can be included as intermediate outcomes. It also guides the analysis of the data; for example, if measures of the theoretical determinants are included, mediation analysis can be used to test hypotheses about causal pathways. This causal modelling approach to intervention development is consistent with the Medical Research Council guidelines on the development and evaluation of complex interventions (Craig et al., 2008), which includes ProActive as a case study.

Third, intervention developers need to be aware of the differences between different theories and should select a theory only after careful consideration of the alternatives available. The ideal theory would (1) be well specified with clear definitions of constructs and clear specifications of the causal relationships among them; (2) have substantial empirical support, including evidence that the putative behavioural determinants do influence behaviour; and (3) specify how the behavioural determinants can be modified. No existing theory satisfies all three criteria, so intervention developers will need to make compromises between the different criteria for theory selection.

Finally, although theories of behaviour change are useful in intervention development, developers need to be aware that there are many aspects of an intervention that such theories cannot inform. For example, theories of behaviour change provide no guidance on the ideal number or schedule of sessions, whether the intervention should be oneto-one or administered in small or large groups or whether audio visual aids should be used. Thus, interventions can never be entirely theory based.

\section{References}

Ajzen, I. 1988: Attitudes, personality, and behavior. Buckingham, UK: Open University Press.

Ajzen, I. 1991: The theory of planned behavior. Organizational Behavior and Human Decision Processes 50, 179-211.

Ajzen, I. 2006: Constructing a TpB questionnaire: Conceptual and methodological considerations. Retrieved 1 September 2010 from www.people.umass.edu/aizen/pdf/ tpb.measurement.pdf

Ajzen, I. and Fishbein, M. 1980: Understanding attitudes and predicting social behavior. Englewood Cliffs, NJ: Prentice Hall.

Barber, N. 2002: Should we consider non-compliance a medical error? Quality and Safety in Health Care 11, 81-84.

Cohen, J. 1992: A power primer. Psychological Bulletin 112, 155-59.

Craig, P., Dieppe, P., Macintyre, S., Michie, S., Nazareth, I. and Petticrew, M. 2008: Developing and evaluating complex interventions: the new Medical Research Council guidelines. British Medical Journal 337, a1655.

Cramer, J.A. 2004: A systematic review of adherence with medications for diabetes. Diabetes Care 27, 1218-24.

Diabetes Prevention Program Research Group. 2002: Reduction in the incidence of type 2 diabetes with lifestyle intervention or metformin. New England Journal of Medicine 346, 393-403.

Farmer, A., Kinmonth, A.L. and Sutton, S. 2006: Measuring beliefs about taking hypoglycaemic medication among people with type 2 diabetes. Diabetic Medicine 23, 265-70. 
Farmer, A.J., Prevost, A.T., Hardeman, W., Craven, A., Sutton, S., Griffin, S.J. and Kinmonth, A.-L., for The Support and Advice for Medication Trial Group. 2008: Protocol for SAMS (Support and Advice for Medication Study): a randomised controlled trial of an intervention to support patients with type 2 diabetes with adherence to medication. BMC Family Practice 9, 20.

Farmer, A.J., Hardeman, W., Hughes, D., Prevost, A.T., Craven, A., Griffin, S., Sutton, S. and Kinmonth, A.-L., on behalf of the Support and Advice for Medication Study Group. SAMS (Support and Advice for Medication Study): randomised controlled trial of a nurse-led consultationbased intervention to support patients' adherence to medication for type 2 diabetes. (Submitted for publication).

Gollwitzer, P.M. and Sheeran, P. 2006: Implementation intentions and goal achievement: a meta-analysis of effects and processes. Advances in Experimental Social Psychology 38, 249-68.

Hagger, M.S. and Orbell, S. 2003: A meta-analytic review of the common-sense model of illness representations. Psychology and Health 18, 141-84.

Hardeman, W., Kinmonth, A.L., Michie, S. and Sutton, S., for the ProActive Project Team. 2009: Impact of a physical activity intervention on cognitive predictors of behaviour among adults at risk of type 2 diabetes (ProActive randomized controlled trial). International Journal of Behavioral Nutrition and Physical Activity 6, 16.

Hardeman, W., Kinmonth, A.L., Michie, S., and Sutton, S., on behalf of the ProActive project team. 2011: Theory of planned behaviour cognitions do not predict self-reported or objective physical activity levels or change in the ProActive trial. British Journal of Health Psychology 16, 135-50.

Hardeman, W., Michie, S., Fanshawe, T., Prevost, A.T., McLoughlin, K. and Kinmonth, A.L. 2008: Fidelity of delivery of a physical activity intervention: predictors and consequences. Psychology \& Health 23, 25-40.

Hardeman, W., Sutton, S., Griffin, S., Johnston, M., White, A., Wareham, N.J. and Kinmonth, A.L. 2005: A causal modelling approach to the development of theory-based behaviour change programmes for trial evaluation. Health Education Research 20, 676-87.

Johnston, M., French, D.P., Bonetti, D. and Johnston, D.W. 2004: Assessment and measurement in health psychology. In Sutton, S., Baum, A. and Johnston, M., editors, The Sage handbook of health psychology. London: Sage.

Kinmonth, A.L., Wareham, N.J., Hardeman, W., Sutton, S., Prevost, A.T., Fanshawe, T., Williams, K.W., Ekelund, U., Spiegelhalter, D. and Griffin, S.J. 2008: Efficacy of a theory-based behavioural intervention to increase physical activity in an at-risk group in primary care (ProActive UK): a randomised trial. Lancet 371, 41-48.

Leventhal, H., Nerenz, D.R. and Steele, D.J. 1984: Illness representations and coping with health threats. In Baum, A., Taylor, S.E. and Singer, J.E., editors, Handbook of psychology and health: social psychological aspects of health, Volume 4. Hillsdale, NJ: Lawrence Erlbaum Associates, Inc., 219-52.

Lewin, K. 1951: Field theory in social science; selected theoretical papers. In Cartwright, D., editor. New York: Harper \& Row.

McLeroy, KR, Bibeau, D, Steckler, A and Glanz, K 1988: An ecological perspective on health promotion programs. Health Education Quarterly 15, 351-77.

Murray, C.J.L. and Lopez, A.D. 1996: The global burden of disease. Harvard, USA: Harvard University Press.

National Institute for Health and Clinical Excellence. 2006: Four commonly used methods to increase physical activity: brief interventions in primary care, exercise referral schemes, pedometers and community-based exercise programmes for walking and cycling. Public Health Intervention Guidance no. 2. London: National Institute for Health and Clinical Excellence.

Ogden, J. 2003: Some problems with social cognition models: a pragmatic and conceptual analysis. Health Psychology 22, 424-28.

Prestwich, A. and Kellar, I. How can the impact of implementation intentions as a behaviour change intervention be improved? Revue Europe?enne de Psychologie Applique?/European Review of Applied Psychology (in press).

Prochaska, J.O. and Velicer, W.F. 1997: The transtheoretical model of health behavior change. American Journal of Health Promotion 12, 38-48.

Rutter, D.R., Steadman, L. and Quine, L. 2006: An implementation intentions intervention to increase uptake of mammography. Annals of Behavioral Medicine $32,127-34$.

Simmons, R.K., van Sluijs E.M., Hardeman W., Sutton S. Griffin S.J., and the ProActive project team. 2010: Who will increase their physical activity? Predictors of change in objectively measured physical activity over 12 months in the ProActive cohort. BMC Public Health 10, 226.

Strecher, V.J. and Rosenstock, I.M. 1997: The health belief model. In Baum, A., Newman, S., Weinman, J., West, R. and McManus, C., editors, Cambridge handbook of psychology, health and medicine. Cambridge: Cambridge University Press, 113-17.

Stroebe, W. 2000: Social psychology and health, second edition. Buckingham, UK: Open University Press.

Sutton, S. 1998: Predicting and explaining intentions and behavior: how well are we doing? Journal of Applied Social Psychology 28, 1317-38.

Sutton, S. 2001: Back to the drawing board? A review of applications of the transtheoretical model to substance use. Addiction 96, 175-86.

Sutton, S. 2004: Determinants of health-related behaviours: theoretical and methodological issues. In Sutton, S., Baum, A. and Johnston, M., editors, The Sage handbook of health psychology. London: Sage, 94-126.

Sutton, S. 2010: Using social cognition models to develop health behaviour interventions: the theory of planned behaviour as an example. In French, D.P., Kaptein, A.A., 
Vedhara, K. and Weinman, J., editors, Health psychology, second edition. Oxford: Blackwell, 122-34.

Weinstein, N.D., Rothman, A.J. and Sutton, S.R. 1998: Stage theories of health behavior: conceptual and methodological issues. Health Psychology 17, 290-99.

West, R. 2005: Time for a change: putting the Transtheoretical (Stages of Change) Model to rest. Addiction 100, 1036-39.

Williams, K., Prevost, A.T., Griffin, S., Hardeman, W., Hollingworth, W., Spiegelhalter, D., Sutton, S., Ekelund, U.,
Wareham, N. and Kinmonth, A.L. 2004: The ProActive trial protocol: randomised controlled trial of the efficacy of a family-based, domiciliary intervention programme to increase physical activity among individuals at high risk of diabetes [ISRCTN 61323766]. BMC Public Health 4, 48.

World Health Organization. 2003: Adherence to long term therapies: evidence for action. Geneva: World Health Organization. 